\title{
Changes of the Alternative Splicing Variants of Human Telomerase Reverse Transcriptase during Gastric Carcinogenesis
}

\author{
Jin-Heng Xu ${ }^{a} \quad$ Yu-Chuan Wang ${ }^{b} \quad$ Xin Geng ${ }^{a} \quad$ Yan-Yun Li ${ }^{a} \quad$ Wei-Ming Zhang ${ }^{a}$ \\ ${ }^{a}$ Department of Biochemistry and Molecular Biology, Tianjin Medical University, and ${ }^{\mathrm{b}}$ Tianjin Institute of \\ Ophthalmology, Tianjin, China
}

\section{Key Words}

Alternative splicing • Precancerous lesion • Gastric cancer •

Telomerase reverse transcriptase

\begin{abstract}
Objective: We attempted to reveal the changes of the human telomerase reverse transcriptase (hTERT) alternative splicing pattern in gastric carcinogenesis. Methods: Three alternative splicing sites $(\alpha, \beta, \gamma)$ were selected and designed PCR primer. The expression of $8 \mathrm{hTERT}$ alternative splicing variants (ASVs) in normal gastric mucosa, precancerous lesions and gastric cancer were detected by seminested RTPCR. The expression of $\beta$-site remaining ASV $\left(\beta^{+}\right.$ASV) in specimens of precancerous lesions and specimens of gastric cancer was detected by SYBER Green real-time PCR. Results: The positive rate of $\alpha^{+} \beta^{+} \gamma^{+}$ASV was significantly higher in gastric cancer than in precancerous lesions and normal mucosa $(94.7$ vs. $40.0 \%$ and $0 \%, p<0.05)$. The positive rates of other ASVs were not different among the 3 groups $(p>0.05)$. The positive rates of $\beta^{+}$ASVs (including $\alpha^{+} \beta^{+} \gamma^{+}$ASV, $\alpha$-deletion ASV, $\gamma$-deletion ASV, $\alpha \gamma$-deletion ASV) were $11.1 \%$ in normal mucosa, $40.0 \%$ in precancerous lesions and $94.7 \%$ in gastric cancer $(p<0.05)$. SYBR Green real-time RT-PCR showed that the expression level of $\beta^{+}$ASV was 6.99 times higher in gastric cancer than in precancerous lesions. Conclusion: hTERT alternative splicing pattern is different dur-
\end{abstract}

\section{KARGER}

Fax +4161306 1234

E-Mail karger@karger.ch

www.karger.com
(C) 2009 S. Karger AG, Basel

1015-2008/09/0761-0023\$26.00/0

Accessible online at:

www.karger.com/pat ing gastric carcinogenesis. $\beta^{+}$ASV was widely expressed in gastric carcinogenesis and may provide some information for diagnosis of gastric cancer or precancerous lesions.

Copyright $\odot 2009$ S. Karger AG, Basel

\section{Introduction}

Gastric cancer is one of the most common malignancies worldwide. Previous studies indicated that there were some changes of gene expression in gastric cancer progression, such as human telomerase reverse transcriptase (hTERT), RAR- $\beta$, p53, CD44, cyclin E, APC, TGF- $\beta$, c-erB2, etc. [1-5]. Telomerase may play a critical role in the rate-limiting step in cancer. Telomerase activity is associated with cancer cell proliferation of gastric carcinogenesis. Therefore, the detection of telomerase activity may be useful for early diagnosis of cancer [6]. Human telomerase RNA is expressed extensively in normal tissues and abnormal tissues. hTERT is a catalytic subunit of the holoenzyme complex and is a rate-limiting factor of telomerase activity. The expression of hTERT is positively correlated with telomerase activity and could represent the telomerase activity [7-10]. Therefore, detecting the expression of hTERT mRNA or protein may be an alternative method of telomerase activity. Alternative splicing exists in the transcription of hTERT 
Table 1. Patients' characteristics

\begin{tabular}{|c|c|c|c|c|c|c|c|}
\hline No. & Sex & Age & Diagnosis & No. & Sex & Age & Diagnosis \\
\hline 1 & $\mathrm{~F}$ & 29 & normal & 29 & $\mathrm{~F}$ & 66 & chronic atrophic gastritis with severe intestinal metaplasia \\
\hline 2 & M & 34 & normal & 30 & M & 72 & chronic atrophic gastritis with moderate intestinal \\
\hline 3 & $\mathrm{~F}$ & 36 & normal & & & & metaplasia \\
\hline 4 & $\mathrm{~F}$ & 28 & normal & 31 & M & 35 & chronic atrophic gastritis with moderate intestinal \\
\hline 5 & $\mathrm{~F}$ & 24 & normal & & & & metaplasia \\
\hline 6 & M & 29 & normal & 32 & $\mathrm{~F}$ & 78 & chronic atrophic gastritis with severe intestinal metaplasia \\
\hline 7 & M & 38 & normal & 33 & M & 65 & chronic atrophic gastritis with moderate intestinal \\
\hline 8 & $\mathrm{~F}$ & 33 & normal & & & & metaplasia \\
\hline 9 & M & 35 & normal & 34 & M & 54 & chronic atrophic gastritis with severe intestinal metaplasia \\
\hline 10 & $\mathrm{~F}$ & 27 & normal & 35 & $\mathrm{~F}$ & 49 & chronic atrophic gastritis with severe atypical hyperplasia \\
\hline 11 & $\mathrm{~F}$ & 33 & normal & 36 & $\mathrm{~F}$ & 33 & chronic atrophic gastritis with severe atypical hyperplasia \\
\hline 12 & M & 38 & normal & 37 & M & 42 & chronic atrophic gastritis with moderate atypical \\
\hline 13 & $\mathrm{~F}$ & 33 & normal & & & & hyperplasia \\
\hline 14 & $\mathrm{~F}$ & 26 & normal & 38 & $\mathrm{~F}$ & 76 & chronic atrophic gastritis with moderate atypical \\
\hline 15 & M & 39 & normal & & & & hyperplasia \\
\hline 16 & M & 40 & normal & 39 & $\mathrm{~F}$ & 59 & adenocarcinoma \\
\hline 17 & $\mathrm{~F}$ & 34 & normal & 40 & $\mathrm{~F}$ & 78 & adenocarcinoma \\
\hline 18 & $\mathrm{~F}$ & 37 & normal & 41 & $\mathrm{~F}$ & 81 & adenocarcinoma \\
\hline \multirow[t]{2}{*}{19} & M & 45 & chronic atrophic gastritis with moderate intestinal & 42 & M & 55 & adenocarcinoma \\
\hline & & & metaplasia & 43 & $\mathrm{M}$ & 62 & adenocarcinoma \\
\hline 20 & M & 39 & chronic atrophic gastritis with severe intestinal metaplasia & 44 & $\mathrm{~F}$ & 79 & adenocarcinoma \\
\hline \multirow[t]{2}{*}{21} & $\mathrm{~F}$ & 44 & chronic atrophic gastritis with moderate intestinal & 45 & M & 72 & adenocarcinoma \\
\hline & & & metaplasia & 46 & $\mathrm{~F}$ & 63 & adenocarcinoma \\
\hline 22 & $\mathrm{~F}$ & 29 & chronic atrophic gastritis with severe intestinal metaplasia & 47 & $\mathrm{M}$ & 59 & adenocarcinoma \\
\hline \multirow[t]{2}{*}{23} & M & 33 & chronic atrophic gastritis with moderate intestinal & 48 & M & 60 & adenocarcinoma \\
\hline & & & metaplasia & 49 & $\mathrm{~F}$ & 67 & adenocarcinoma \\
\hline \multirow[t]{2}{*}{24} & M & 56 & chronic atrophic gastritis with moderate intestinal & 50 & M & 68 & adenocarcinoma \\
\hline & & & metaplasia & 51 & $\mathrm{~F}$ & 79 & adenocarcinoma \\
\hline 25 & $\mathrm{~F}$ & 60 & chronic atrophic gastritis with severe intestinal metaplasia & 52 & $\mathrm{M}$ & 65 & adenocarcinoma \\
\hline \multirow[t]{2}{*}{26} & M & 69 & chronic atrophic gastritis with moderate intestinal & 53 & $\mathrm{~F}$ & 58 & adenocarcinoma \\
\hline & & & metaplasia & 54 & $\mathrm{M}$ & 69 & adenocarcinoma \\
\hline 27 & M & 45 & chronic atrophic gastritis with severe intestinal metaplasia & 55 & $\mathrm{~F}$ & 60 & adenocarcinoma \\
\hline \multirow[t]{2}{*}{28} & $\mathrm{~F}$ & 67 & chronic atrophic gastritis with moderate intestinal & 56 & $\mathrm{~F}$ & 55 & adenocarcinoma \\
\hline & & & metaplasia & 57 & $\mathrm{M}$ & 79 & adenocarcinoma \\
\hline
\end{tabular}

and is associated with telomerase activity [11-13]. Three alternatively spliced sites of hTERT pre-mRNA were selected in this study. The primers were designed according to the three spliced sites $\alpha, \beta, \gamma$. We detected the expression of hTERT alternative splicing variants (ASVs) in normal gastric mucosa, precancerous lesions and gastric cancer and we hope that it may provide some information for the diagnosis of precancerous lesions or gastric cancer.

\section{Materials and Methods}

Tissue Samples

All the specimens were obtained from Tianjin Medical University General Hospital (from March to June 2006). The samples included 18 normal gastric mucosa specimens, 20 precancerous lesions of gastric specimens and 19 gastric cancer specimens (detailed information is shown in table 1). After obtaining the specimens, they were quickly put into liquid nitrogen and stored at $-80^{\circ} \mathrm{C}$.

\section{Total RNA Isolation and RT-PCR}

Frozen specimens were quickly put into a freezing microtone $\left(-30^{\circ} \mathrm{C}\right)$ and were sectioned while frozen. All the frozen sections were put on slides and stained with toluidine blue. Of every two sections, one section was selected and stained with HE in order to find the position of target cells. Sections stained with toluidine blue were put on an inverted microscope and target cells found according to HE sections. Then needles of a sterile syringe $(1 \mathrm{ml})$ were used to remove connective tissue and other nontarget cells. Sections with target cells were quickly stored at $-80^{\circ} \mathrm{C}$.

Total RNAs were extracted from the frozen sections with target cells using TRIzol ${ }^{\mathrm{TM}}$ (Invitrogen Life Technologies). The final RNA preparations were quantified by absorbance analysis at 260 $\mathrm{nm}$ and tested by $1 \%$ agarose gel electrophoresis. $2 \mu \mathrm{g}$ of total RNA was reverse transcribed in a $25-\mu \mathrm{l}$ reaction volume using oligodT (Promega) and M-MLV (Promega) reverse transcriptase and the reaction buffer provided in the Reverse Transcription kit (Promega). 


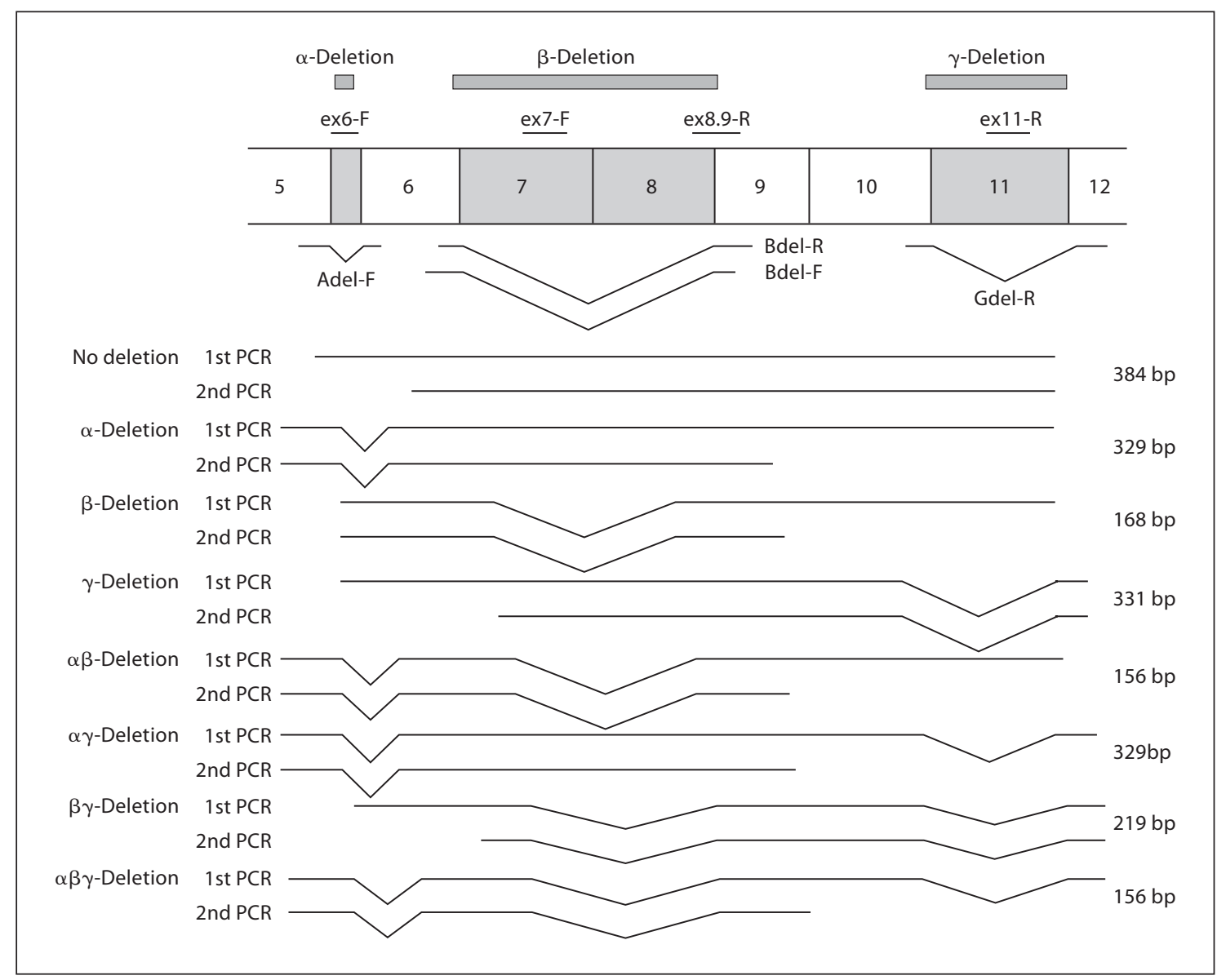

Fig. 1. Three alternative splicing sites lead to 8 ASVs. The $\alpha$-site deletion causes a 36 -base deletion and the $\beta$-site deletion results in a 182-base deletion in exon 7 and 8 . The $\gamma$-site deletion induces a 189-base deletion in exon 11. The Adel-F, ex6-F, ex7-F and Bdel-F primers were forward primers, and ex11-R, ex8.9-R, Bdel-R and Gdel-R were reverse primers.

Table 2. Primers for hTERT mRNA

\begin{tabular}{lll}
\hline Name & Primer & Position \\
\hline ex6-F & 5'-ATG TGA CGG GCG CGT ACG AC-3' & $2191-2210$ \\
ex11-R & 5'-GGA AGT TCA CCA CTG TCT TCC GC-3' & $2756-2778$ \\
Adel-F & 5'-CTG CAG GAG ACC AGC CCG C-3' & $2167-2186,2223-2228$ \\
Gdel-R & 5'-GTA GGA CTT GCC CCT GAT GCG-3' & $2702-2710,2900-2913$ \\
ex7-F & 5'-CTG AGC TGT ACT TTG TCA AGG ACA GG-3' & $2394-2412$ \\
ex8.9-R & 5'-AGG TCC GGG CAT AGC TGA GGA AG-3' & $2511-2531$ \\
Bdel-F & 5'-GCC TTC AAG AGC CAC GTC CTA CG-3' & $2327-2342,2525-2531$ \\
Bdel-R & 5'-GCA CTG GAC GTA GGA CGT GGC T-3' & $2336-2342,2525-2540$ \\
\hline
\end{tabular}

Seminested RT-PCR Testing hTERT ASVs

The primers of hTERT ASVs are shown in table 2 and figure 1. All the primers were designed according to hTERT (AF0181670) alternative splicing sites [14]. The initial step of RTPCR was 2 min at $95^{\circ} \mathrm{C}$ for incubation. Cycles $(n=30)$ consisted of a 30 -second denaturation phase at $95^{\circ} \mathrm{C}$, a 30 -second annealing at $60^{\circ} \mathrm{C}$ and a 40 -second extension at $72^{\circ} \mathrm{C}$. The final phase was at $72^{\circ} \mathrm{C}$ incubation for $10 \mathrm{~min}$. The positive products of seminested RT-PCR were reclaimed and sequenced. 
Fig. 2. The position of primers for GADPH and $\beta^{+}$ASV.

Table 3. Primers for GAPDH (NM_2046) and $\beta^{+}$ASV

\begin{tabular}{|c|c|c|c|c|c|c|c|c|}
\hline \multicolumn{1}{|c|}{$\beta$-Deletion site } & \multicolumn{1}{c|}{$\gamma$-Deletion site } \\
\hline Exon 5 & 6 & 7 & 8 & 9 & 10 & 11 & 12 \\
\hline \multicolumn{3}{|c|}{$\overline{\beta^{+}-\mathrm{F}}$} & $\overline{\beta^{+}-\mathrm{R}}$ \\
\hline
\end{tabular}

\begin{tabular}{llc}
\hline Name & Sequence & Position \\
\hline$\beta^{+}-\mathrm{F}$ & 5'-AGG TCA TCG CCA GCA TCA TCA AAC-3' $^{\prime}$ & $2236-2259$ \\
$\beta^{+}-\mathrm{R}$ & 5'-ACG GCT GGA GGT CTG TCA AGG TAG-3' $^{\prime}$ & $2347-2370$ \\
GAPDH-F & $5^{\prime}$-GGT GAA GGT CGG AGT CAA CGG A-3' & $111-132$ \\
GADPH-R & 5'-GAG GGA TCT CGC TCC TGG AAG A-3' $^{\prime}$ & $329-350$ \\
\hline
\end{tabular}

Table 4. Positive rates of hTERT ASVs in gastric carcinoma, precancerous lesions and normal gastric mucosa by seminested RT-PCR

\begin{tabular}{lclllllll}
\hline Groups & $\alpha^{+} \beta^{+} \gamma^{+}$ & $\begin{array}{l}\alpha \text {-Deletion } \\
\text { ASV, } \%\end{array}$ & $\begin{array}{l}\beta \text {-Deletion } \\
\text { ASV, } \%\end{array}$ & $\begin{array}{l}\gamma \text {-Deletion } \\
\text { ASV, } \%\end{array}$ & $\begin{array}{l}\alpha \beta \text {-Deletion } \\
\text { ASV, } \%\end{array}$ & $\begin{array}{l}\beta \gamma \text {-Deletion } \\
\text { ASV, } \%\end{array}$ & $\begin{array}{l}\alpha \gamma \text {-Deletion } \\
\text { ASV, } \%\end{array}$ & $\begin{array}{l}\alpha \beta \gamma \text {-Deletion } \\
\text { ASV, \% }\end{array}$ \\
\hline Normal gastric mucosa & $0.0(0 / 18)$ & $16.7(3 / 18)$ & $72.2(13 / 18)$ & $0.0(0 / 18)$ & $33.3(6 / 18)$ & $11.1(2 / 18)$ & $5.6(1 / 18)$ & $0.0(0 / 18)$ \\
Precancerous lesions & $40.0(8 / 20)$ & $15.0(3 / 20)$ & $95.0(19 / 20)$ & $5.0(1 / 20)$ & $50.0(10 / 20)$ & $25.0(5 / 20)$ & $25.0(5 / 20)$ & $0.0(0 / 20)$ \\
Gastric cancer & $94.7(18 / 19)$ & $10.5(2 / 19)$ & $100.0(19 / 19)$ & $5.3(1 / 19)$ & $42.1(8 / 19)$ & $15.8(3 / 19)$ & $10.5(2 / 19)$ & $0.0(0 / 19)$ \\
\hline
\end{tabular}

Numbers are given in parentheses. ${ }^{*} \mathrm{p}<0.05$.

SYBER Green Real-Time RT-PCR Assay of $\beta^{+}$ASVs

$\beta^{+}$ASVs ( $\beta$-site remaining ASVs) were amplified by RT-PCR. The primers are shown in table 3 and figure 2. RT-PCR products include all $\beta$-site-reserved ASVs. Six $\beta^{+}$ASV precancerous lesion specimens and 6 gastric cancer specimens were selected and identified by SYBER Green real-time RT-PCR. The real-time PCR data were analyzed using the $2^{-\Delta \Delta \mathrm{CT}}$ relative quantization method according to the manufacturer's directions.

\section{Results}

\section{Results of Seminested RT-PCR}

The results of hTERT ASVs are shown in table 4 and figure 3. $\alpha^{+} \beta^{+} \gamma^{+}$ASV was absent in normal gastric mucosa, and its positive rate was higher in gastric cancer than that in precancerous lesions $(\mathrm{p}<0.05)$. $\beta$-Deletion ASVs were expressed in normal gastric mucosa, precancerous lesions and gastric cancer, and the positive rates of $\beta$-deletion ASVs, respectively, were 72.2, 95.0 and $100 \%$. $\alpha \beta \gamma$-deletion ASVs were not detected in this study and another 5 kinds of ASVs had no statistical significance among normal gastric mucosa, precancerous lesions and gastric cancer $(\mathrm{p}>0.05)$. The positive rates of $\beta$-site deletion ASV expression (including $\beta$-deletion ASV, $\alpha \beta$ deletion ASV, $\beta \gamma$-deletion ASV) were 83.3, 95.0 and $100.0 \%$, respectively, in normal gastric mucosa, precancerous lesions and gastric cancer, but the differences had no statistical significance in the above three groups. The positive rates of $\beta^{+}$ASV expression (including $\alpha^{+} \beta^{+} \gamma^{+}$ ASV, $\alpha$-deletion ASV, $\gamma$-deletion ASV, $\alpha \gamma$-deletion ASV) were, respectively, 11.1, 40.0 and $94.7 \%$, and the differences in the three groups were statistically significant $(\mathrm{p}<0.05)$. The results of all hTERT ASV sequences were identical with the target sequences.

Result of SYBER Green Real-Time RT-PCR of $\beta^{+}$ASV

$\beta^{+}$ASV was not detected in normal gastric mucosa by PCR. Of 20 precancerous lesion specimens, 6 expressed 


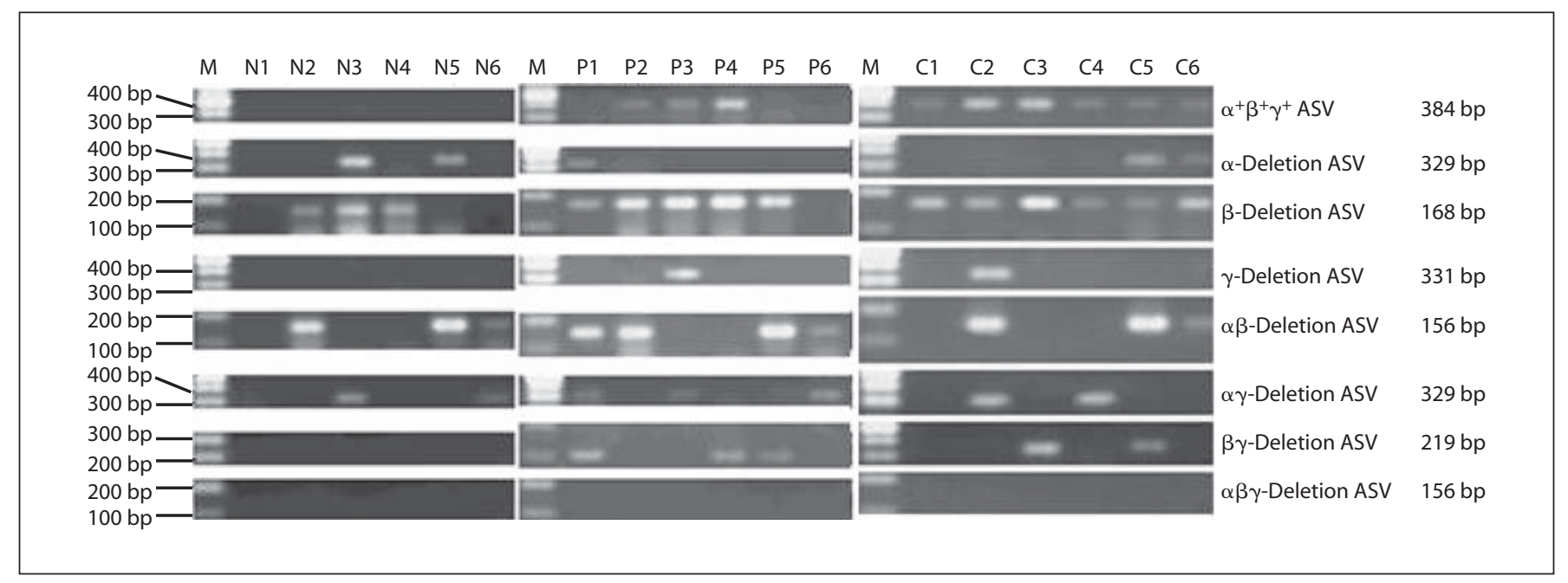

Fig. 3. Representative gel pictures of seminested RT-PCR of hTERT ASVs. $\mathrm{N}=$ Normal gastric mucosa; $\mathrm{P}=$ precancerous lesions; $\mathrm{C}$ = gastric cancer.

$\beta^{+}$ASV, while of 19 gastric cancer specimens, 16 expressed $\beta^{+}$ASV. The differences were statistically significant $(\mathrm{p}<0.05) . \beta^{+}$ASV in precancerous lesions and gastric cancer were selected and performed using fluorescence real-time RT-PCR. The expression level of $\beta^{+}$ASV was 6.99 times higher in gastric cancer than that in precancerous lesions using the $2^{-\Delta \Delta C T}$ method.

\section{Discussion}

mRNA splicing is a fundamental biological process involved in the expression of genes, and about a third of human genes are thought to be alternatively spliced [15, 16]. Some alternatively spliced variants have been associated with a subset of human diseases [17-20]; however, the pathological importance of ASVs in most human diseases is still uncertain. Telomerase is activated in a variety of malignant tumors [6] and is thought to be necessary for cellular immortality and carcinogenesis. Telomerase activity was detected in gastritis patients with chronic atrophy, patients with intestinal metaplasia, dysplasia patients and gastric cancer patients. Telomerase is expressed during the early stage of gastric carcinogenesis [21]. Regulation of telomerase activity is a complex process involving transcriptional control, posttranslational modification, positively and negatively acting factors and alternative splicing $[22,23]$. The core telomerase consists of two key components, the catalytic unit hTERT and an RNA template (hTER) [24]. Telomerase activity has been shown to correlate well with the expression level of hTERT [25-27]. The hTERT gene includes 16 exons and 15 introns [28]. The hTERT protein contains one conserved reverse transcriptase motif $A$ and seven reverse transcriptase motifs. hTERT $m$ RNA has at least 13 alternative splicing sites, including 3 deletion sites $(\alpha, \beta, \gamma)$ and 10 insertion sites. In our study 3 deletion sites were selected to study the alternative splicing patterns in gastric carcinogenesis.

$\alpha$-Deletion ASV and $\gamma$-deletion ASV result from inframe deletions of exonic sequences in exon 6 and 11, respectively. The translation products of these two ASVs lost a small part of hTERT protein. Researchers speculated that these two ASVs appear to be inhibitors of telomerase activity $[10,29]$. In our study, we found that the positive rates of $\alpha$-deletion ASV and $\gamma$-deletion ASV had no statistical significance, and this result is consistent with a previous report [30]. We could not confirm that these two ASVs are inhibitors of telomerase activity. This may be because these two ASVs downregulated telomerase activity in a dose-dependent manner, but our study could not quantify them.

We observed that $\alpha^{+} \beta^{+} \gamma^{+}$ASV transcript (unspliced ASV) was present in $40.0 \%$ of precancerous lesions and $94.7 \%$ of gastric cancer; however, it was absent in normal gastric mucosa. The difference was significant. The result was in agreement with that reported by Barclay et al. [30]. A previous study showed that expression of normal fulllength hTERT correlates well with telomerase activity [31]. Telomerase activity was found to be higher in gastric 
tumor samples than in normal tissue [32]. Barclay et al. [30] found there was a significant positive correlation between telomerase activity and hTERT mRNA expression in normal gastric mucosa, but there was no significant correlation between telomerase activity and levels of hTERT mRNA in gastric cancer. However, our study did not show the relationship of telomerase activity and fulllength hTERT.

We observed that $\beta$-deletion ASV was widely expressed in normal gastric mucosa, precancerous lesions and gastric cancer. We also found that other ASVs $(\alpha \beta$ deletion ASV, $\beta \gamma$-deletion ASV, $\alpha \gamma$-deletion ASV) were lower expressed in the three groups and the differences were nonsignificant. This may be because these ASVs lost many motifs of hTERT gene or because of the introduction of early stop codons. We did not detect $\alpha \beta \gamma$-deletion ASV and this may be because it is unstable or easily degraded. Twelve cases of $\beta^{+}$ASV (including 6 cases with precancerous lesions and 6 cases with gastric cancer specimens) were tested by real-time fluorescence quantitative RT-PCR. The results showed that the expression level of $\beta^{+}$ASV was 6.99 times higher in gastric cancer than in precancerous lesions. The pathology reports of these specimens showed that $4 / 20$ precancerous lesions were chronic atrophic gastritis with moderate or severe atypical hyperplasia and 16/20 precancerous lesions were chronic atrophic gastritis with moderate to severe intestinal metaplasia. We found that $\beta^{+}$ASV was expressed in all dysplasia specimens, but only a small number of intestinal metaplasia lesions (2/16) expressed $\beta^{+}$ASV. Therefore, we assume that the expression of $\beta^{+}$ASV may reflect the evolution of gastric cancer from intestinal metaplasia to dysplasia. The expression of $\beta^{+}$ASV was higher in gastric cancer than in precancerous lesions. We guess that the increase in $\beta^{+}$ASV may be involved in the carcinogenesis of precancerous lesions.

In conclusion, our study confirmed that hTERT alternative splicing patterns are different in gastric carcinogenesis. We assume that the increase in $\beta^{+}$ASV may be involved in the carcinogenesis of precancerous lesions. The hTERT alternative splicing pattern is more complicated. In our study we only selected three splicing sites. If other new sites are found, further research will be necessary.

\section{Acknowledgments}

This work was supported by the National Natural Science Foundation of China (grant No. 39770298).

\section{References}

1 Vauhkonen M, Vauhkonen H, Sipponen P: Pathology and molecular biology of gastric cancer. Best Pract Res Clin Gastroenterol 2006;20:651-674

$\checkmark 2$ Yasui W, Sentani K, Motoshita J, Nakayama $\mathrm{H}$ : Molecular pathobiology of gastric cancer. Scand J Surg 2006;95:225-231.

3 Anderson C, Nijagal A, Kim J: Molecular markers for gastric adenocarcinoma: an update. Mol Diagn Ther 2006;10:345-352.

4 Hofler H, Becker KF, Keller G: Molecular carcinogenesis of the upper gastrointestinal tract. Verh Dtsch Ges Pathol 2006;87:123129.

5 Oliveira C, Seruca R, Carneiro F: Genetics, pathology and clinics of familial gastric cancer. Int J Surg Pathol 2006;14:21-33.

-6 Hiyama E, Hiyama K: Clinical utility of telomerase in cancer. Oncogene 2002;21: 643-649.

7 Price DK, Figg WD: hTert-immortalized endothelial cells: an important new research tool. Cell Cycle 2004;3:789.

8 Janknecht R: On the road to immortality: hTERT upregulation in cancer cells. FEBS Lett 2004;564:9-13.
9 Saldanha SN, Andrews LG, Tollefsbol TO: Analysis of telomerase activity and detection of its catalytic subunit, hTERT. Anal Biochem 2003;315:1-21.

10 Kirkpatrick KL, Mokbel K: The significance of human telomerase reverse transcriptase (hTERT) in cancer. Eur J Surg Oncol 2001; 27:754-760.

11 Li G, Ma S, Zhao C, Wang H, Shi Y, Xu G: Expression of telomerase gene hTRT and its clinical significance in gastric cancer. Tumor 2003;23:47.

$\$ 12$ Hisatomi H, Ohyashiki K, Ohyashiki JH, Nagao K, Kanamaru T, Hirata H, Hibi N, Tsukada Y: Expression profile of a gammadeletion variant of the human telomerase reverse transcriptase gene. Neoplasia 2003;5: 193-197.

13 Saeboe-Larssen S, Fossberg E, Gaudernack G: Characterization of novel alternative splicing sites in human telomerase reverse transcriptase (hTERT): analysis of expression and mutual correlation in mRNA isoforms from normal and tumour tissues. BMC Mol Biol 2006;29:7-26.
14 Nagao K, Katsumata K, Aizawa Y, Saito N, Hirata H, Sasaki H, Yamamoto S, Hikiji K, Koiwa T, Hisatomi H: Differential alternative splicing expressions of telomerase reverse transcriptase in gastrointestinal cell lines. Oncol Rep 2006;11:127-131.

15 Hanke J, Brett D, Zastrow I, Aydin A, Delbruck S, Lehmann G, Luft F, Reich J, Bork P: Alternative splicing of human genes: more the rule than the exception? Trends Genet 1999;15:389-390.

16 Mironov AA, Fickett JW, Gelfland MS: Frequent alternative splicing of human genes. Genome Res 1999;9:1288-1293.

-17 Stallings-Mann ML, Ludwiczak RL, Klinger KW, Rottman F: Alternative splicing of exon 3 of the human growth hormone receptor is the result of an unusual genetic polymorphism. Proc Natl Acad Sci USA 1996;93. 12394-12399.

18 Liu W, Qian C, Francke U: Silent mutation induces exon skipping of fibrillin-1 gene in Marfan syndrome. Nat Genet 1997;16:328329. 
19 Siffert W, Posskopf D, Siffert G, Busch S, Moritz A, Erbel R, Sharma AM, Ritz E, Wichmann HE, Jakobs KH, Horsthemke B: Association of human G-protein beta3 subunit variant hypertension. Nat Genet 1998; 18:45-48.

-20 Hisatomi H, Kohno N, Wakita K, Nagao K, Hirata H, Hikiji K, Harada S: A novel alternatively spliced variant with a deletion of $52 \mathrm{bp}$ in exon 6 of the progesterone receptor gene is frequently observed in breast cancer. Int J Cancer 2003; 105:182-185.

-21 Yang SM, Fang DC, Luo YH, Lu R, Battle PD, Liu WW: Alterations of telomerase activity and terminal restriction fragment in gastric cancer and its premalignant lesions. J Gastroenterol Hepatol 2001;16:876-882.

-22 Pilch B, Allemand E, Facompre M, Bailly C, Riou JF, Soret J, Tazi J: Specific inhibition of serine- and arginine-rich splicing factors phosphorylation, spliceosome assembly, and splicing by the antitumor drug NB-506. Cancer Res 2001;61:6876-6884.

23 Hisatomi H, Nagao K, Komatsu H: Quantification of telomerase activity in human liver tissues by fluorescence-based TRAP analysis. Hepatol Res 1997;7:35-42.
24 Cohen S, Graham M, Lovrecz G, Bache N, Robinson P, Reddel R: Protein composition of catalytically active human telomerase from immortal cells. Science 2007;315:1850 1853.

25 Cong YS, Wright WE, Shay JW: Human telomerase and its regulation. Microbiol Mol Biol Rev 2002;66:407-425.

26 Toshikuni N, Nouso K, Higashi T, Nakatsukasa H, Onishi T, Kaneyoshi T, Kobayashi Y, Kariyama K, Yamamoto K, Tsuji T: Expression of telomerase-associated protein 1 and telomerase reverse transcriptase in hepatocellular carcinoma. Br J Cancer 2000;82: 833-837.

27 Ito H, Kyo S, Kanaya T, Takakura M, Inoue M, Namiki M: Expression of human telomerase subunits and correlation with telomerase activity in urothelial cancer. Clin Cancer Res 1998;4:1603-1608.

28 Cong YS, Wen J, Bacchetti S: The human telomerase catalytic subunit hTERT: Organization of the gene and characterization of the promoter. Hum Mol Genet 1999;8:137142 .
29 Colgin LM, Wilkinson C, Englezou A, Kilian A, Robinson MO, Reddel RR: The hTERT alpha splice variant is a dominant negative inhibitor of telomerase activity. Neoplasia 2000;2:426-432.

30 Barclay JY, Morris AG, Nwokolo CU: hTERT mRNA partially regulates telomerase activity in gastric adenocarcinoma and adjacent normal gastric mucosa. Dig Dis Sci 2005;50: 1299-1303.

31 Saebøe-Larssen S, Fossberg E, Gaudernack G: Characterization of novel alternative splicing sites in human telomerase reverse transcriptase (hTERT): analysis of expression and mutual correlation mRNA isoforms from normal and tumor tissues. BMC Mol Biol 2006;7:26.

32 Gümüş-Akay G, Ünal AE, Bayar S, Karadayi K, Elhan AH, Sunguroğlu A, Tükün A: Telomerase activity could be used as a marker for neoplastic transformation in gastric adenocarcinoma: but it does not have a prognostic significance. Genet Mol Res 2007;6: 41-49. 